\title{
La Ruptura Prematura de Membranas
}

\author{
HOSPITAL MATERNO INFANTIL 1983 a 1984
}

\author{
Dres. Luis Vicente López A.*, Laura Rojas R.**, Víctor Rodríguez M.*** \\ Jacinto Sánchez A.****.
}

\section{INTRODUCCION}

La Ruptura Prematura de Membranas es un acontecimiento de frecuente ocurrencia en obstetricia y de no menor importancia por la repercusión que representa para la madre y su producto.

Su incidencia varía mucho no solamente en las diferentes maternidades, sino dentro de éstas en las diferentes épocas, lo cual nos hace pensar en la complejidad de las causas que la originan. Así, por ejemplo para Bollar (1936) es de $1.7 \%$, Sunders (1938) es de $13 \%$, J.K. Nerutte (1942) $3.4 \%$, Taylor (1961) $6.3 \%$, Berty (1967) $7.8 \%$ y entre nosotros Velasco (1963) $0.66 \%$ (9).

Desde el punto de vista etiológico se invocan muchos factores, pero cualquiera que sean ellos, las complicaciones parecen estar relacionadas siempre con la infección en la madre y el sindrome de dificultad respiratoria e infección en el producto, como las más frecuentes y graves $(1,3,6)$.

* Profesor Asociado. Departamento de Ginecología y Obstetricia - Facultad de Medicina - Universidad Nacional.

** Profesor Asistente. Departamento de Ginecología y Obstetricia - Facultad de Medicina - Universidad Nacional.

*** Especialista en Ginecología y Obstetricia - Instituto Materno Infantíl.

**** Instructor Asociado. Departamento de Ginecología y Obstetricia - Facultad de Medicina - Universidad Nacional.
Si tenemos en cuenta que la Ruptura Prematura de Membranas es un accidente de mucha importancia en obstetricia, es sorprendente encontrarnos hoy con una divergencia tan notoria en cuanto a su manejo y tratamiento. No se sabe si esto es debido a multicasualidad etiológica en el desarrollo natural de la entidad en los diferentes medios y grupos poblacionales o es que no tenemos un método óptimo de asistencia aún.

El Hospital Materno Infantil no es precisamente la excepción a este problema. Es por eso que la inquietud nos ha llevado a estudiar el fenómeno y sus consecuencias en nuestro medio.

\section{MATERIAL Y METODOS}

Durante el período de un año (1o. agosto 1983 a 31 de julio de 1984) asistieron al Hospital Materno Infantil de Bogotá 11.048 pacientes para la atención del parto, de las cuales 566 presentaban ruptura prematura de membranas.

Teniendo en cuenta que las más frecuentes y graves complicaciones en la Ruptura Prematura de Membranas son la sépsis y el parto pretérmino con sus consecuencias, el síndrome de dificultad respiratoria en el neonato, hemos planeado los siguientes parámetros de manejo.

1. Las pacientes con embarazo a término o cerca del término, igual o mayor de 36 semanas, en 
cuyo caso se considera que el producto no va a tener dificultad desde el punto de vista madurez pulmonar y solamente se puede complicar desde el punto de vista de la infección, tanto la madre como el producto; se desembarazaron en el menor tiempo posible, según las normas del servicio hasta ahora establecidas y de acuerdo con las condiciones particulares del caso.

2. Las pacientes con embarazos menores de 27 semanas de gestación, consideramos que los productos no son viables en nuestro medio, su comportamiento es el de un aborto en curso y la desembarazamos de inmediato.

3. Las pacientes con 27 a 35 semanas de gestación las hemos considerado como productos viables pero con un alto riesgo de infección si se prolonga el embarazo, en busca de madurez pulmonar y de sindrome de dificultad respiratoria si no se hace.

Para su manejo las hemos dividido en dos grupos A y B.

Grupo A (control). Se desembarazan en el menor tiempo posible de acuerdo a normas del servicio.

Grupo B (de estudio). Se dejan en observación por 48 a 72 horas, buscando una aceleración de la madurez pulmonar y luego se desembarazan según indicaciones propias de cada caso.

A este último grupo se le practicó ecografía y Test de Clements, para confirmar edad gestacional y madurez pulmonar respectivamente. Se tomaron cultivos de líquido amniótico y de secreción vaginal. A la mayoría se les practicó monitoría fetal externa.

\section{RESULTADOS}

FRECUENCIA: durante el período de estudio hubo 11.048 partos de los cuales 566 presentaban Ruptura Prematura de Membranas; 495 en embarazos a término o cerca de término (igual o mayor a 36 semanas de gestación) y 71 embarazos pretérmino de 27 a 35 semanas (Tabla 1 ).

Características Generales Maternas: en cuanto a la edad se encontraron 324 pacientes entre los 20
TABLA 1

FRECUENCIA DE LA RUPTURA PREMATURA DE MEMBRANAS EN EL HOSPITAL MATERNO INFANTIL EN EL PERIODO DE AGOSTO DE 1983 Y EL 30 DE JULIO DE 1984

Población Porcentaje

Partos Atendidos

11.048

Total de R P M

$566 \quad 5.18 \%$

R P M entre 27 y $35 \mathrm{~S}$

$71 \quad 0.64 \%$

R P M igual o mayor de $35 \mathrm{~S}$ 495

$4.48 \%$

y 30 años, un caso de paciente menor de 15 años y mayores de 35 años, 55 casos. (Fig. 1).

FIG. 1

EDAD

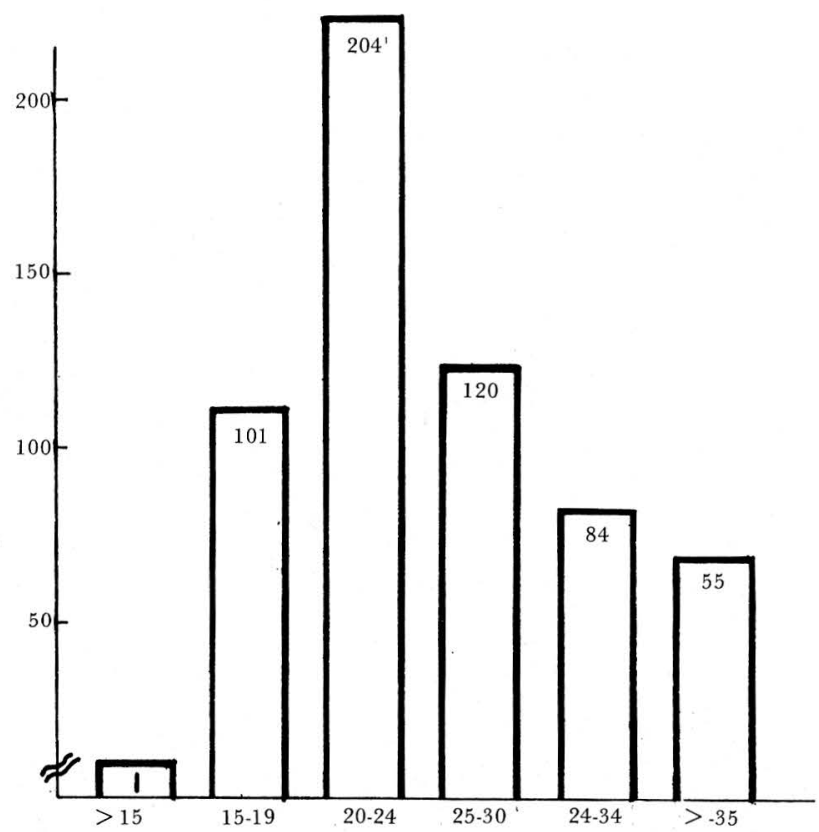

Paridad

La Ruptura Prematura de Membranas con embarazo a término se presentó en 290 nulíparas y 
245 multíparas. En el embarazo pretérmino se presentaron 21 casos de nulíparas y 50 multíparas (Fig. 2).

FIG. 2

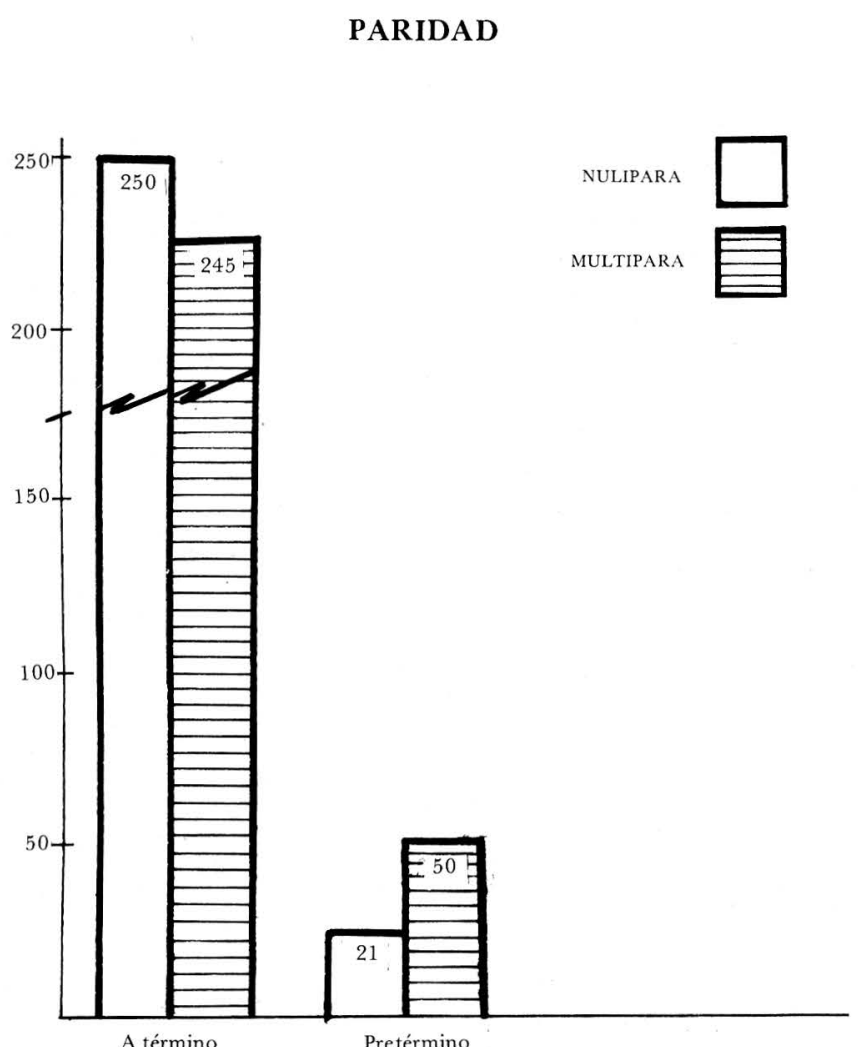

\section{Control Prenatal:}

431 Asistieron a control prenatal y 135 no tuvieron asistencia prenatal.

\section{Antecedentes Ginecoobstétricos:}

Se observaron 94 pacientes con flujo genital que no fue tratado durante el embarazo. Tres pacientes tenían antecedente de operación de Manchester, de las cuales 2 presentaron parto pretérmino. 46 Pacientes tenían antecedente de Ruptura Prematura de Membranas en embarazos anteriores; 58 antecedente de aborto y 56 antecedente de cesárea (Tabla 2).
TABLA 2

RUPTURA PREMATURA DE MEMBRANAS ANTECEDENTES PERSONALES MEDICOS

$\begin{array}{lrr}\text { Preeclampsia } & \text { II } & (1.91 \%) \\ \text { Diabetes } & 4 & (0.69 \%) \\ \text { Asma } & 4 & (0.69 \%) \\ \text { Hepatitis } & 4 & (0.69 \%) \\ \text { Estado Gripal } & 5 & (0.87 \%) \\ \text { Total } & 28 & (4.88 \%)\end{array}$

Ginecológicos:

Leucorrea

94
1
3 $\quad\left(\begin{array}{r}(16.40 \%) \\ (0.17 \%)\end{array}\right.$

O. Manchester $\left(\begin{array}{l}0.17 \%) \\ (0.52 \%)\end{array}\right.$

Obstétricos:

$\begin{array}{lrr}\begin{array}{lrr}\text { R. P. M. } \\ \text { Abortos }\end{array} & 446 & (8.02 \%) \\ \text { Cesáreas } & 58 & (10.12 \%) \\ \text { Infección Urinaria } & 56 & (9.77 \%) \\ \text { Control Prenatal } & 431 & (75.53 \%) \\ \end{array}$

Diagnóstico de Admisión:

De las 566 pacientes a quienes se les diagnosticó Ruptura Prematura de Membranas, 496 tenían embarazo de 36 o más semanas; 71 entre 27 a 35 semanas de gestación, 291 presentaban un tiempo de Ruptura menor de 12 horas, 152 entre 13 y 24 horas, 107 entre 25 y 72 horas y 16 más de 72 horas; 218 pacientes llegaron en trabajo de parto; 18 con signos francos de infección y 6 presentaban óbito fetal (Fig. 3-4).

\section{Comportamiento de la Ruptura Prematura} de Membranas:

Hubo 217 pacientes que iniciaron trabajo de parto espontáneo así: 201 con embarazo de 36 o más semanas de gestación y 16 embarazos entre 27 a 35 semanas. 
FIGURA 3

DIAGNOSTICO DE ADMISION

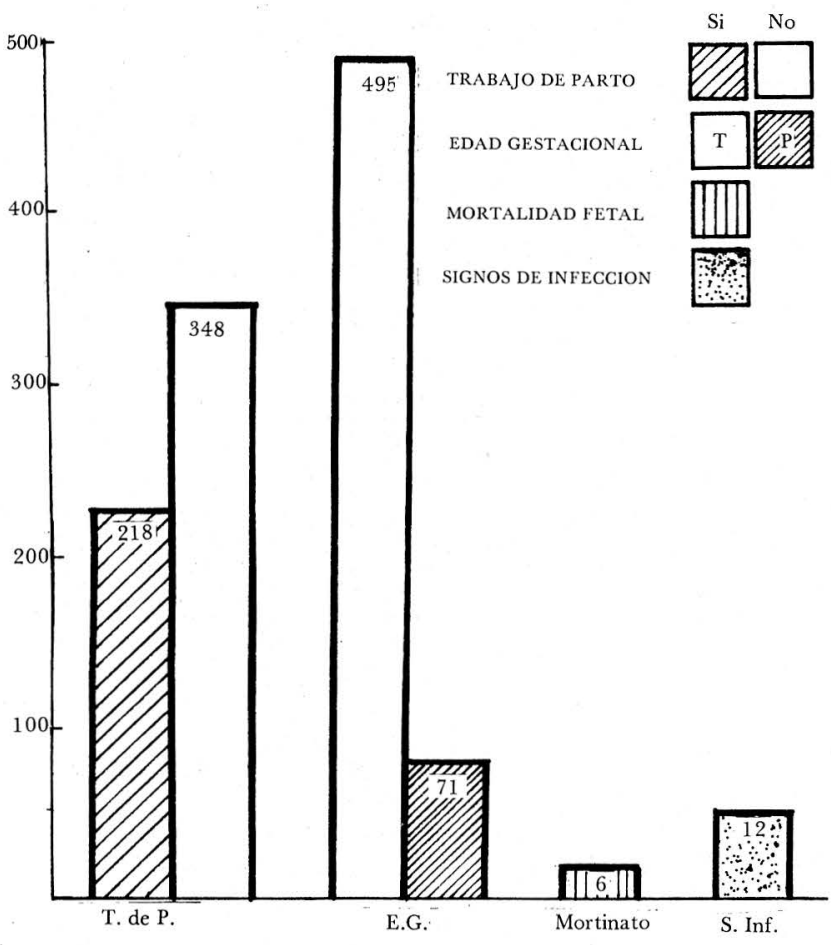

FIGURA 4

RUPTURA DE MEMBRANA AL INGRESO

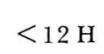

$13-24 \mathrm{H}$
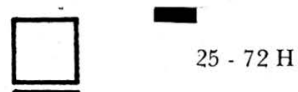

$<73 \mathrm{H}$

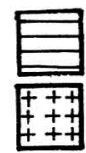

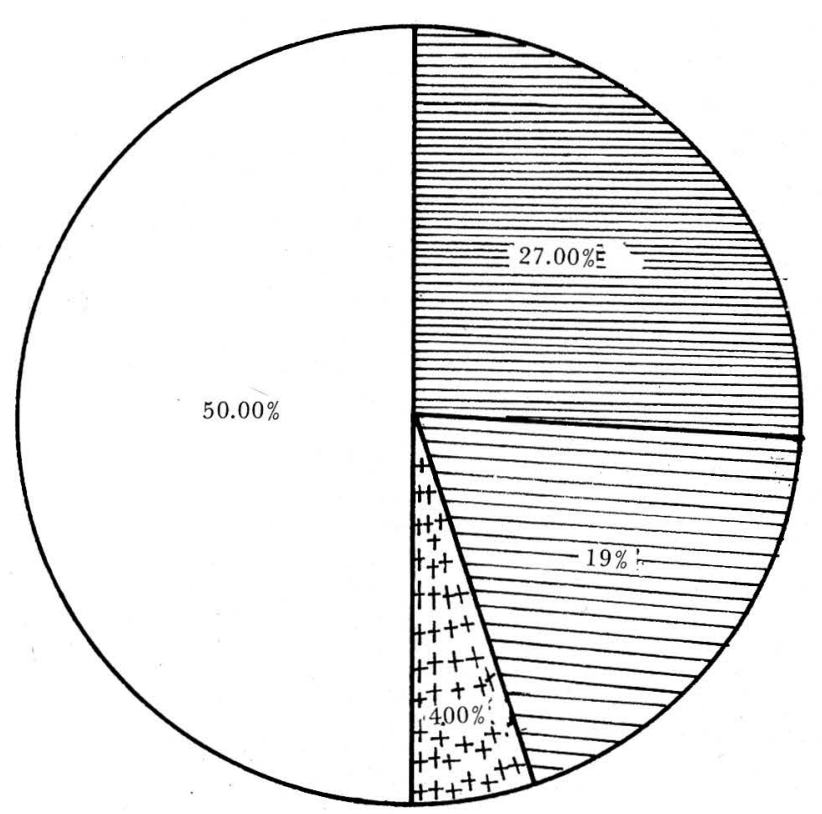

Se practicó inducción del trabajo de parto en 239 , de las cuales 205 con embarazo de 36 o más semanas y 34 entre 27 a 35 semanas. Se practicaron 77 cesáreas al ingreso de las pacientes sin trabajo de parto. (Fig. 5).

FIGURA 5

\section{INICIACION DEL TRABAJO DE PARTO}
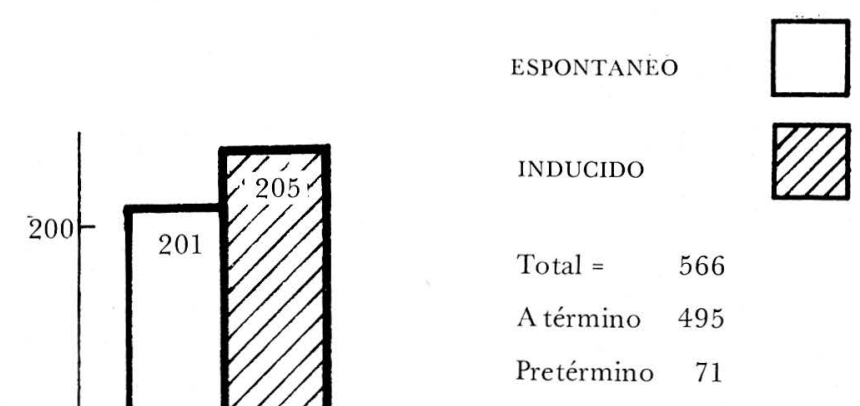

Pretérmino 71

Tiempo de Latencia:

Con tiempo de latencia menor de 12 horas hubo 228 pacientes; entre 13 y 24 horas 161 ; entre 25 a 72 horas 153 y 29 pacientes con más de 72 horas. Entre las pacientes con embarazos de 27 a 35 semanas el tiempo de latencia fue el siguiente: menos de 12 horas 17 pacientes; de 13 a 24 horas 9 pacientes; de 25 a 72 horas 32 pacientes y más de 72 horas 15 pacientes. Las pacientes que llegaron en trabajo de parto presentaron tiempo de latencia menor de 12 
horas 78 , de 13 a 24 horas 55 , entre las 25 a 72 horas 32 y mayor de 72 horas 3 pacientes. Total 168 pacientes.

Duración del Trabajo de Parto:

415 presentaron trabajo de parto menor de 12 horas y 25 mayor de 12 horas.

\section{Modalidad del Parto:}

379 presentaron parto vía vaginal y a 187 se les practicó cesárea. Se distribuyeron de acuerdo a la edad gestacional en la siguiente forma:

Embarazos de 36 o más semanas, vía vaginal 335 y cesáreas 160 . Embarazos de 27 a 35 semanas, vía vaginal 44 pacientes, cesáreas 27 .

FIGURA 6

INDICACIONES DE GESAREA

EN EMBARAZO A TERMINO

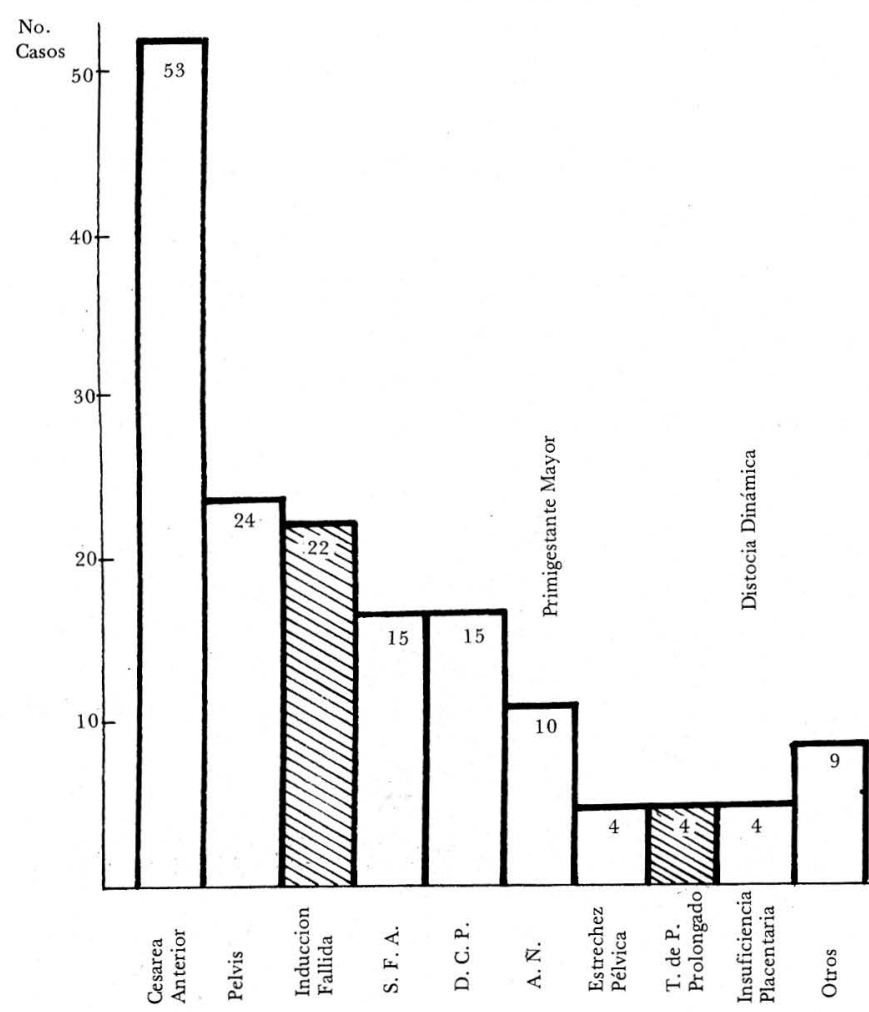

De éstas últimas en el grupo control hubo 19 partos vaginales y 7 cesáreas. En el grupo estudio hubo 14 vía vaginal y 14 cesáreas. (Fig. 7).

\section{Indicaciones de la Cesarea:}

Se practicaron 187 cesáreas; 160 en embarazos de más de 36 semanas y en embarazos de 27 a 35 semanas 27. Por inducción fallida 22 casos, sufri. miento fetal agudo 15 casos, distocia dinámica 4 casos. El resto no tenía una relación directa con la Ruptura Prematura de Membranas. (Fig. 6-7).

\section{Uso de Antibióticos:}

De las pacientes que tuvieron parto vaginal 104 recibieron antibióticos y de las cesárea 146, para un total de 250 pacientes.

FIGURA 7

EMBARAZO PRETERMINO:

INDICACION DE CESAREA

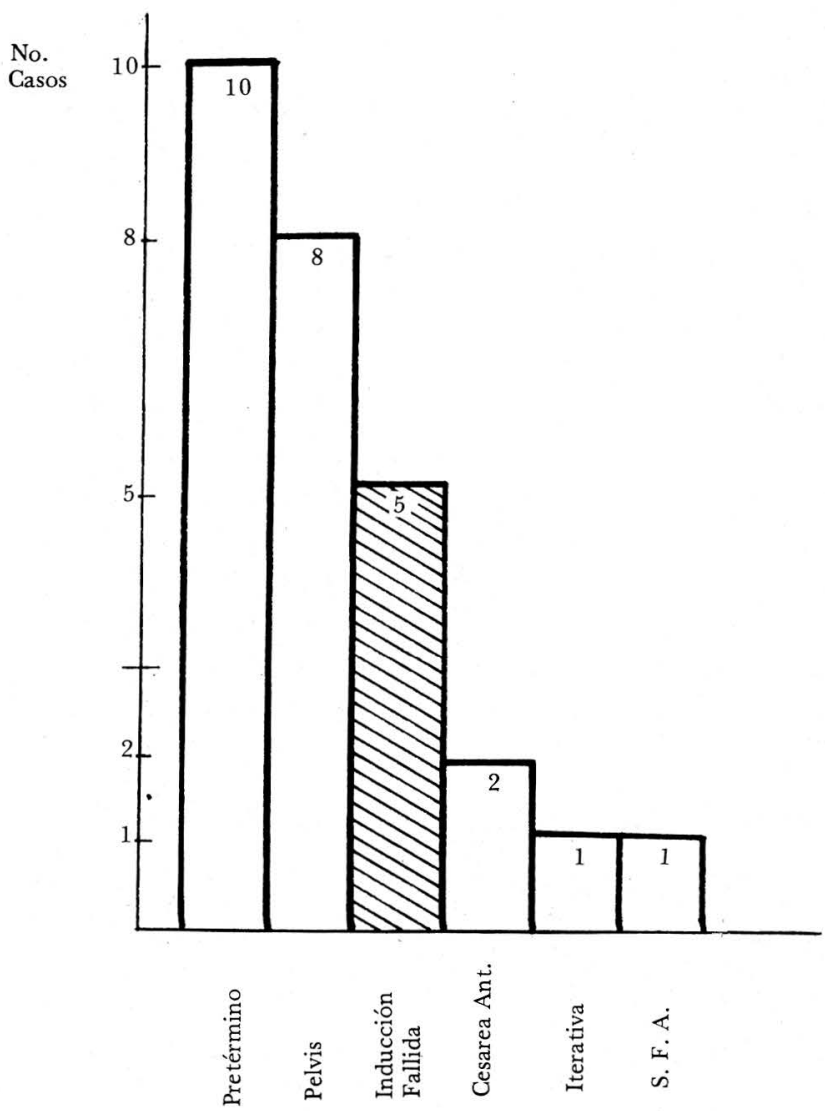




\section{COMPLICACIONES DE LA RUPTURA PREMATURA DE MEMBRANAS}

Entre las complicaciones que presentaron las pacientes con Ruptura Prematura de Membranas tenemos la infección, ya sea, ante parto o después de éste, en la siguiente forma:

\section{Corioamnionitis:}

Se presentaron 30 casos, de los cuales 18 ingresaron con signos francos de infección y se manifestaron intra hospitalariamente 12 casos; 21 tenían embarazo de 36 o más semanas y entre 27 a 35 hubo 9 . Estas pacientes presentaron un tiempo de latencia promedio de 35 horas las de 36 o más semanas y de 68 las 27 a 35 semanas. Todas recibieron antibióticos antes y después del parto.

Las complicaciones puerperales en este grupo fueron: un caso de endometritis, un caso de infección urinaria y uno de morbilidad febril.

Las complicaciones fetales y neonatales fueron: 10 sufrimientos fetales agudos; una muerte intraparto; 3 presentaron sepsis y un pretérmino, sindrome de dificultad respiratoria. Se presentaron 5 muertes neonatales tempranas por anoxia intraparto $\mathrm{y}$ uno por sepsis. La mortalidad neonatal fue de $23 \%$.

\section{Morbilidad Infecciosa Postparto}

Entre las pacientes que tenían 36 o más semanas de embarazo se presentaron 9 casos de endometritis, 7 en las de parto por cesárea y en el parto vaginal. El tiempo de latencia promedio fue de 13 horas, 4 habían recibido antibióticos antes de manifestarse la infección. El resto lo recibió después. Todas se recuperaron sin más complicaciones. En las pacientes con embarazo pretérmino se presentaron 2 endometritis ambas en el grupo control; en el grupo estudio no hubo morbilidad materna. (Tabla 3).

\section{Morbilidad por Sufrimiento Fetal Agudo}

Se presentaron 28 casos de sufrimiento fetal agudo, 17 en los embarazos de 36 o más semanas y 11 en los de 27 a 35 semanas.

En el grupo pretérmino control se presentaron 3 sufrimientos fetales agudos y fallecieron los 3 . En
TABLA 3

\section{MORBILIDAD MATERNA INFECCIOSA}

\begin{tabular}{|c|c|c|c|c|c|c|c|c|}
\hline \multicolumn{9}{|c|}{ Morbilidad General a Término Control Estudio } \\
\hline & $\begin{array}{l}\text { Cesa- } \\
\text { rea. }\end{array}$ & $\begin{array}{l}\text { Vagi- } \\
\text { nal. }\end{array}$ & $\begin{array}{l}\text { Cesa- } \\
\text { rea. }\end{array}$ & $\begin{array}{l}\text { Vagi- } \\
\text { nal. }\end{array}$ & $\begin{array}{l}\text { Cesa- } \\
\text { rea. }\end{array}$ & $\begin{array}{l}\text { Vagi- } \\
\text { nal. }\end{array}$ & $\begin{array}{l}\text { Cesa- } \\
\text { rea. }\end{array}$ & $\begin{array}{l}\text { Vagi- } \\
\text { nal. }\end{array}$ \\
\hline Endometriosis & 164 & 91 & 8 & 2 & 2 & 0 & 0 & 0 \\
\hline $\begin{array}{l}\text { Abceso de } \\
\text { Pared }\end{array}$ & 124 & 0 & 3 & 0 & & & & \\
\hline Infección Urinaria & 34 & 3 & 6 & 0 & & & & \\
\hline Peritonitis & 8 & 0 & 0 & 0 & & & & \\
\hline Sepsis & 5 & 1 & 0 & 0 & & & & \\
\hline $\begin{array}{l}\text { Respiratorias } \\
\text { Altas }\end{array}$ & 4 & 8 & 0 & 0 & & & & \\
\hline Flebitis & 6 & 2 & 1 & 1 & & & & \\
\hline $\begin{array}{l}\text { Infección en la } \\
\text { Episiorrafia }\end{array}$ & 0 & 40 & 0 & 5 & & & & \\
\hline $\begin{array}{l}\text { Respiratoria } \\
\text { Bajas }\end{array}$ & 15 & 3 & 0 & 0 & & & & \\
\hline $\begin{array}{l}\text { Morbilidad } \\
\text { Febril }\end{array}$ & 0 & 0 & 5 & 3 & & & & \\
\hline Total & 347 & $19.09 \%)$ & 159 & .72) 2 & $3(14.3$ & $7 \%) 11$ & $(3.26 \%)$ & $2(15.38 \%) 000$ \\
\hline Población & 1.8 & $8 i 7$ & 923 & & 160 & & 335 & $\begin{array}{llll}13 & 20 & 14 & 14\end{array}$ \\
\hline
\end{tabular}

el grupo pretérmino estudio se presentó un caso que falleció.

\section{Morbilidad por Sindrome de Dificultad Respiratoria}

En los recién nacidos de 36 o más semanas se presentaron 12 casos de sindrome de dificultad respiratoria transitoria.

En los recién nacidos de 27 a 35 semanas se presentaron 36 casos de sindrome de dificultad respiratoria, de los cuales en el grupo control hubo 16 casos y en el grupo estudio 12. De éstos fallecieron 9 en el grupo control y 4 en el grupo de estudio.

De los recién nacidos que presentaron sindrome de dificultad respiratoria en el grupo control 6 desarrollaron Membrana Hialina comprobada radiológicamente y fallecieron 4. Del grupo de estudio la presentaron 4 y todos fallecieron y a ninguno se le practicó autopsia.

\section{Morbilidad Infecciosa en el Recién Nacido}

La sepsis se presentó en 21 recién nacidos; hubo 12 en los neonatos de 36 o más semanas, de los 
cuales fallecieron 3. En los recién nacidos de 27 a 35 semanas se presentaron 9 de los cuales fallecieron 5. Del grupo control 4 y fallecieron 3 y del grupo de estudio se presentó un caso el cual falleció. (Tabla 4).

TABLA 4

MORBILIDAD POR INFECCION EN LA R. P. M.

\begin{tabular}{|llcc|}
\hline R. N. & Sepsis & $\%$ & Mortalidad \\
\hline General & 76 & $0.7 \%$ & 60 \\
$\begin{array}{l}\text { A término } \\
\text { Pretérmino }\end{array}$ & $12(3)$ & $2.4 \%$ & 3 \\
$\begin{array}{l}\text { Control } \\
\text { Pretérmino } \\
\text { Estudio }\end{array}$ & $8(4)$ & $15.38 \%$ & $4(3)$ \\
\hline
\end{tabular}

\section{Mortalidad Neonatal Temprana en la RPM}

Se practicó un seguimiento hasta los 7 días y se presentaron 25 muertes.

FIGURA 8

\section{MUERTES NEONATALES}

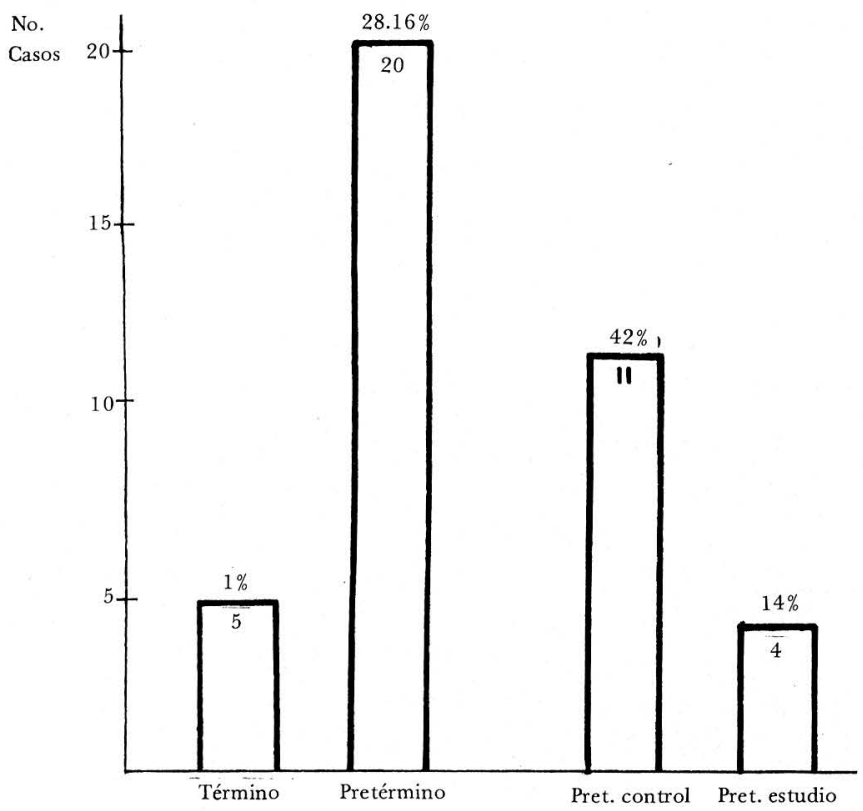

En los recién nacidos mayores de 36 semanas hubo 5 muertes, 3 por infección y 2 por hipoxia neonatal severa.

En el embarazo entre 27 a 35 semanas hubo 20 muertes de las cuales 5 fallecieron por sepsis y 15 por sindrome de dificultad respiratoria.

En el grupo control 11 muertes; 3 por sepsis y el resto por sindrome de dificultad respiratoria.

En el grupo estudio hubo 4 muertes, 3 por sindrome de dificultad respiratoria y uno por sepsis. (Fig. 8).

FIGURA 8

MORTALIDAD DEL RECIEN NACIDO PRETERMINO

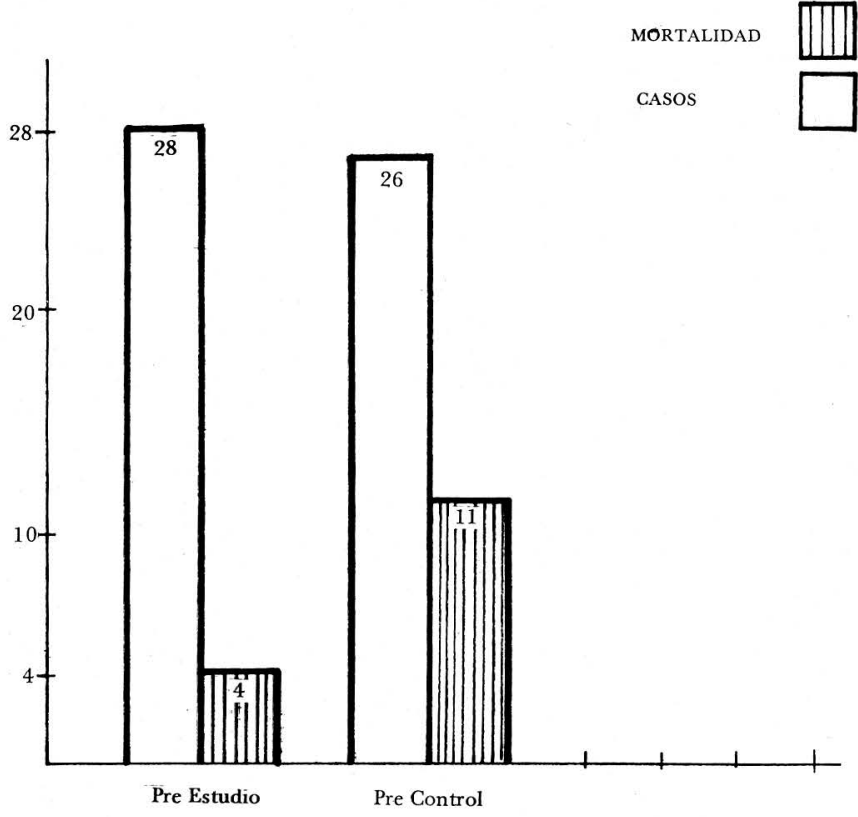

DISCUSION

Frecuencia

La frecuencia de la Ruptura Prematura de Membranas en el Instituto Materno Infantil de Bogota, en el período del 1o. de agosto de 1983 al 31 de julio de 1984 , fue de un $5 \%$ con un $0.64 \%$ para el embarazo pretérmino (27 a 35 semanas). (Tabla 1$)$.

\section{Características Generales Maternas}

No se presentó diferencia entre las multíparas y las nulíparas en cuanto a frecuencia en el parto a 
término siendo la relación de 1:1. En cambio en los embarazos pretérmino se presentó mayor frecuencia en las multíparas con una relación de 3:1. (Fig. 2).

Cuando se analizó la frecuencia de Ruptura Prematura de Membranas en relación con: infertilidad previa, cesárea anterior, aborto anterior, enfermedades generales, alteración o infección de vías urinarias, hemorragia durante el embarazo y trastornos hipertensivos no hubo diferencias significativas. (Tabla 2).

\section{Diagnóstico de Admisión}

Se observó que la mayor frecuencia de Ruptura Prematura de Membranas se presenta en el embarazo a término o cerca de término un $87.5 \%$ y solamente un $12,5 \%$ en el pretérmino.

A pesar del riesgo que representa la RPM, se observó que el $50 \%$ de las pacientes consultó al Hospital después de 12 horas de haberse presentado ésta, con un $3 \%$ de corioamnionitis la cual pudo haberse evitado. Durante el prenatal se instruye a la paciente al respecto.

Un $1 \%$ de las pacientes presentaron mortinatos a los cuales no se les pudo practicar autopsia y por consiguiente no quedó establecida la causa de muerte.

\section{Comportamiento de la Ruptura Prematura de Membranas}

Un $40 \%$ de las pacientes presentó trabajo de parto espontáneo, $41 \%$ de las a término y $24.6 \%$ de las pretérmino.

E1 $43 \%$ de las pacientes se indujeron con ocitocina, así: $42 \%$ a término y $52 \%$ pretérmino.

E1 17\% de las que también llegaron en preparto se les practicó cesárea por indicación obstétrica desde el ingreso.

El comportamiento de la Ruptura Prematura de Membranas en cuanto al tiempo de iniciación del trabajo de parto es el siguiente:

E1 79\% inició trabajo de parto antes de las 24 horas, el $19 \%$ entre las 24 y 72 horas y el $2 \%$ después de las 72 horas.
El tiempo de latencia en el embarazo a término fue menor de 24 horas en el $73 \%$; entre 24 a 72 horas el $24.5 \%$ y después de las 72 horas un $2.5 \%$.

En el embarazo pretérmino el tiempo de latencia se distribuyó así:

Menor de 24 horas en el 38\%; entre 24 y 72 horas un $43.5 \%$ y mayor de 72 horas un $21 \%$.

En resumen, el $80 \%$ de las pacientes inician trabajo antes de las 24 horas. El período de latencia aumenta a medida que disminuye el tiempo de gestación, lo cual le daría tiempo al $60 \%$ de los embarazos pretérmino de intentar madurez pulmonar.

Se observó una mayor frecuencia de cesáreas en las pacientes con Ruptura Prematura de Membranas $(32 \%)$ ya que, el índice para la población del Hospital es de $16 \%$ en este período. La inducción fallida fue la causa en el 14\% de los casos; el sufrimiento fetal agudo en el 9\% y la dilatación estacionaria en el $2.5 \%$.

En el embarazo pretérmino la frecuencia de la cesárea fue todavía mayor $38 \%$, de las cuales fueron $37 \%$ por pretérmino de bajo peso, el $18 \%$ por inducción fallida, el $29 \%$ por pelvis y el resto por otras causas.

En relación con los antibióticos no se observó un criterio definido en su uso. En general se aplicaron después de las 12 horas de Ruptura Prematura de Membranas.

Las principales complicaciones de la Ruptura Prematura de Membranas son la infección, tanto materna como del producto y el sindrome de dificultad respiratoria para el neonato pretérmino.

\section{Corioamnionitis}

La frecuencia de corioamnionitis en la Ruptura Prematura de Membranas fue de $5 \%$ encontrándose en relación directa con el tiempo de latencia mayor de 24 horas en el embarazo a término, lo cual se encuentra de acuerdo con los hallazgos de Taylor en Norteamérica y de Velasco en nuestro medio. En cuanto al embarazo pretérmino el tiempo de latencia aumentó el riesgo de infección después de las 72 horas, siendo el índice de infección en este grupo 
de $10 \%$ ésto es 5 veces menor que el síndrome de dificultad respiratoria que fue de $50 \%$.

Se observó un alto índice de sufrimiento fetal agudo en las pacientes con corioamnionitis, una muerte intraparto y un $23 \%$ de mortalidad neonatal temprana, lo cual nos alerta en un mejor control prenatal y la monitorización de estas pacientes durante el trabajo de parto, si queremos disminuír la mortalidad en estos casos. En cambio en la madre se observó una baja morbilidad puerperal con este manejo.

La conducta de desembarazar la paciente en el menor tiempo posible, en el embarazo a término, nos permitió observar una morbilidad de $17.5 \%$, menor de los índices establecidos en el hospital que es del $21 \%$. En relación al riesgo de infección la cesárea la aumentó. Sin embargo, se anota que no se presentó mortalidad materna durante este período.

En relación con el parto pretérmino el índice de infección fue del 14\% y el hecho de aumentar el tiempo de latencia de 48 a 72 horas, en procura de una mayor madurez pulmonar, no aumentó el riesgo de infección en estas pacientes.

En ninguna de las 28 pacientes del grupo de estudio se presentó morbilidad postparto.

La morbilidad fetal estuvo relacionada con el sufrimiento fetal agudo en $4.7 \%$ y con la infección in útero en $5 \%$. A pesar de que el SFA es alto en nuestro medio y muy posiblemente está relacionado con la compresión del cordón, se amerita un mejor seguimiento de estos casos para establecer con exactitud la causa.

El sindrome de dificultad respiratoria se presentó en un $2 \%$ en el embarazo mayor de 36 semanas, en el embarazo pretérmino fue de $50 \%$. En el grupo control en un $60 \%$ y en el grupo en estudio $43 \%$, lo cual nos permite decir que el SDR, es menos frecuente cuando se aumenta el tiempo de latencia, sin que esta diferencia haya sido estadísticamente significativa.

El índice de infección neonatal en el hospital es de $0.7 \%$ y la Ruptura Prematura de Membranas aumentó el riesgo 5 veces. En el embarazo pretérmino hubo un $12.5 \%$. Analizando por aparte la fre- cuencia en el grupo control fue de $15 \%$ y en el grupo de estudio $3.5 \%$, vemos que el tiempo de latencia de 48 a 72 horas no aumentó el índice de infección en estos neonatos.

Se analiza la mortalidad neonatal temprana y la sepsis y se obtienen los siguientes datos:

En el hospital se presentó una mortalidad neonatal temprana de 4\%. La Ruptura Prematura de Membranas no aumentó el riesgo en forma global 4.4\%. La mortalidad neonatal temprana en el grupo de estudio fue 3 veces menor que en el grupo control, lo cual es estadísticamente significativo $(\mathrm{P}=$ 0.49 ).

La mortalidad por sindrome de dificultad respiratoria en el grupo de estudio disminuyó a la mitad en relación con el grupo control. La mortalidad por sepsis fue 3 veces menor en el grupo en estudio que en el grupo control.

\section{CONCLUSIONES}

1. La frecuencia de Ruptura Prematura de Membranas en el Hospital Materno Infantil de Bogotá, durante el período 1o. de agosto de 1983 al 31 de julio de 1984 , fue de $5 \%$ y para el embarazo pretérmino de $0.67 \%$.

2. La Ruptura Prematura de Membranas es más frecuente en el embarazo a término (87.5\%) que en el embarazo pretérmino $(12.5 \%)$.

3. La Ruptura Prematura de Membranas aumentó al doble el índice de cesáreas (30\%) establecido para el mismo período (16\%). Siendo la inducción fallida el 13\%, el SFA 9\% y la dilatación estacionaria las causas.

4. La compresión del cordón como causa de sufrimiento fetal agudo no se pudo establecer claramente por falta de un seguimiento adecuado durante el trabajo de parto y de la consignación de estos datos después de la cesárea.

5. Las mayores complicaciones de la RPM fueron la infección, tanto materna como del producto $\mathrm{y}$ el sindrome de dificultad respiratoria en el neonato. 
6. La frecuencia de corioamnionitis fue de $5 \%$. La infección en el embarazo a término estuvo relacionada con el tiempo de latencia mayor de 24 horas.

7. La morbilidad por SDR en el neonato pretérmino a pesar de que se redujo en un $33 \%$ en el grupo de estudio no fue estadísticamente significativa.

8. La infección tanto materna como fetal, no aumentó al menos hasta las 72 horas de tiempo de latencia en el grupo de estudio.

9. La mortalidad neonatal en el grupo control fue 3 veces mayor, el SDR 2 veces mayor que en el grupo de estudio. Lo cual nos permite suponer que el prolongar el tiempo de latencia (al menos por 72 horas) en procura de una mayor madurez pulmonar, es beneficioso para el neonato en la Ruptura Prematura de Membranas con embarazo pretérmino.

10. A consecuencia de la Ruptura Prematura de Membranas no se observó mortalidad materna.

\section{RESUMEN}

Durante el período del 1o. de agosto de 1983 al 31 de julio de 1984, se practicó seguimiento a las pacientes con Ruptura Prematura de Membranas y se estableció su frecuencia, la morbimortalidad materno fetal y neonatal, relacionando el tiempo de latencia con el sindrome de dificultad respiratoria y la sepsis en el embarazo pretérmino.

Se presentó una frecuencia de Ruptura Prematura de Membranas de $5 \%$ con embarazo pretérmino de $0.67 \%$.

La mayor frecuencia de Ruptura Prematura de Membranas se presentó en el embarazo a término $87.5 \%$ y un $12.5 \%$ en pretérmino. No se observó di- ferencia en cuanto a paridad, siendo igual en multíparas que en nulíparas, en el embarazo a término pero en el embarazo pretérmino fue más frecuente en las multíparas 3: 2.

El índice de cesárea se ve aumentado por la Ruptura Prematura de Membranas 30\% comparado con el establecido para el mismo período que fue de $16 \%$.

Las mayores complicaciones de la Ruptura Prematura de Membranas fueron la infección, tanto materna como fetal y el sindrome de dificultad respiratoria del neonato pretérmino.

La corioamnionitis se manisfestó en el 5\% de las pacientes.

La morbilidad postparto materna en el embarazo a término fue de $14 \%$ en la cesárea y de $3 \%$ en el parto vaginal. En el embarazo pretérmino $14 \%$ para la cesárea y no hubo morbilidad en el parto vaginal. El grupo de estudio al cual se dejó mayor tiempo de latencia en procura de madurez pulmonar no presentó morbilidad materna postparto.

La morbilidad del recién nacido fue del $50 \%$ para el sindrome de dificultad respiratoria y del 10\% para la infección, en los neonatos pretérmino. El grupo de estudio se redujo a $33 \%$ el SDR con relación al grupo control. La infección no se vió aumentada en el grupo de estudio.

La morbilidad neonatal fue de 4.4 para la Ruptura Prematura de Membranas. La mortalidad neonatal en el grupo de estudio disminuyó 3 veces en relación con el grupo control $(\mathrm{P}=0.49)$.

Se concluye que en el embarazo pretérmino con Ruptura Prematura de Membranas se debe procurar la madurez pulmonar antes de desembarazar la paciente disponiendo de un tiempo de latencia no menor de 48 horas, ya que durante éste no se vió aumentado el riesgo de infección.

\section{BIBLIOGRAFIA}

1. MEAD P. B.: Asistencia de la paciente con Ruptura Prematura de Membranas. Clínicas de Perinatología 2: 245, 1980.
2. Velasco CH. A.: Ruptura Prematura de Membranas. Rev. Col. Obstetricia y Ginecología 63. 1967. 
3. LEDGER W. J.: Ruptura Prematura de Membranas e infección materno-fetal. Clínicas Obstétricas y Ginecológicas 2: 337, 1979.

4. MILlER J. M.: PUPKIN M. J. and HILL G. B.: Bacterial Colonization From amniotic fluid with intact fetal membranes. Am I Obst-Gynecol 136: 796, 1980.

5. MILlER J. M., HILl G., WELT S. I. and PUPKIN M. J.: Bacterial colonization of amniotic fluid in the presence of ruptured membranes. Am I Obst-Gynecol 137: 451, 1980.

6. Views and Reviews: Departamento de Obst y Ginecología de la Escuela de Medicina y Hospital Monte Sinaí N. Jersey. Manejo de la Ruptura Prematura de Membranas: 1. Cerca de Término. Obs \& Gynecol 32: 855, 1968.

7. DAIKOKU N. H., KALTREIDER F., JHON T. R. and SIMMONS M. A.: Premature Rupture of Membranes and Preterm Labor: Neonatal and perinatality Risks. Obst \& Ginecol 58: 336, 1981.

8. LIGGIN G. C.: A controlled trial of antepartum glucocorticoid treatment for prevention of the respiratory distress syndrome in premature infants. Pediatrics 50: 515, 1972.

9. BAUER CR, STERN L., COLLE E.: Prolonged Rupture of membranes associated with a decreased indicence of respiratory distress syndrome. Pediatrics 53: 7, 1974.
10. SACHS M., BAKER T. H.: Spontaneaus premature rupture of the membranes. Am I Obst Gynecol 97: 88, 1967.

11. LEDGER W. J., NORMAN M., GEe C. L., LEWIS W. P.: Bacteremia on an obstetric-ginecologic service. Am I Obst Gynecol 121: 205, 1975.

12. MILler J. M., PUPKIN M. J., CREnSHAW C. JR.: Premature labor and premature rupture of membranes. Am I ObstGinecol 132: 1978.

13. RONALD S. GIBBS. Premature Rupture of the Membranes Obstetrics and Gynecology Vol 60 número 61982 Diciembre.

14. RICHARD L. NUCYE. Factors That predispos to premature Rupture of the fetal membranes. Obstetrics and Gynecology Vol. 60 No. 1,julio 1982.

15. RICHARD L. Bir Ro witz. Results of a Management Protocol for Premature Rupture of the Membranes. Obstetrics and Gynecology Vol 60 No. 3, septiembre 1982.

16. JOAN C. NILSON. Premature Rupture of Membranes Prior to consequences of non intervention. Obstetrics and Gynecology Vol 60 No. 5, noviembre 1982. 